Real Analysis Exchange

Vol. 23(2), 1997-1998, pp. 567-570

Harvey Rosen, Department of Mathematics, University of Alabama, Tuscaloosa, AL 35487 e-mail: hrosen@mathdept.as.ua.edu

\title{
AN ALMOST CONTINUOUS NONEXTENDABLE FUNCTION
}

\begin{abstract}
An example is constructed under the Continuum Hypothesis showing that almost continuity and the Strong Cantor Intermediate Value Property do not imply extendability. This answers a question in [8]. Results about stationary sets are given for the class of extendable functions from $I$ into $I$, where $I=[0,1]$.
\end{abstract}

In 1955, John Nash introduced the idea of a "connectivity" function $f$ : $X \rightarrow Y$ by requiring the graph of its restriction $f_{\mid C}$ to be a connected subset of $X \times Y$ for each connected subset $C$ of $X$ [5]. A discontinuous connectivity function can arise from a differential equation. For example, for $x \neq 0$, the curve

$$
y= \begin{cases}\sin \frac{1}{x} & \text { if } x \neq 0 \\ 0 & \text { if } x=0\end{cases}
$$

is a solution of $x^{4} y^{\prime \prime}+2 x^{3} y^{\prime}+y=0$ (to which $t=\frac{1}{x}$ transforms $\ddot{y}+y=0$ ).

A connectivity function $f: I \rightarrow I$ is extendable if there exists a connectivity function $F: I^{2} \rightarrow I$ such that $F(x, 0)=f(x)$ for all $x \in I$. A function $f: I \rightarrow I$ is called almost continuous if each open neighborhood of the graph of $f$ in $I^{2}$ contains the graph of a continuous function on $I$. In [8], it was shown that an extendable function $f: I \rightarrow I$ has the Strong Cantor Intermediate Value Property (SCIVP), which means that whenever $f(x) \neq f(y)$ and $E$ is a Cantor set between $f(x)$ and $f(y)$, there exists a Cantor set $B$ lying between $x$ and $y$ such that $f(B) \subset E$ and $f_{\mid B}$ is continuous. It was also shown that there exists an almost continuous function $f: I \rightarrow I$ which has a perfect road at every point and does not have the SCIVP. The following result answers the question asked there.

Key Words: almost continuous function, extendable connectivity function, the Strong Cantor Intermediate Value Property, stationary set

Mathematical Reviews subject classification: 26A15, 54C08

Received by the editors August 20, 1997 
Theorem 1. Under $\mathrm{CH}$, there exists an almost continuous function $f: I \rightarrow I$ which has the SCIVP but is not extendable.

Proof. Let $\mathcal{D}=\left\{A_{\alpha}: \alpha \in A\right\}$ denote the collection of all dense $G_{\delta}$-subsets of $I$ and $\mathcal{X}=\left\{K_{\alpha}: \alpha \in A\right\}$ denote the collection of all minimal blocking sets of $I^{2}$. A "minimal blocking set" of $I^{2}$ is a smallest closed subset of $I^{2}$ that misses some function from $I$ into $I$ but meets every continuous function from $I$ into $I$ [4]. We may suppose $A$ is well ordered so that each $\alpha$ in $A$ is preceded by less than $c$-many elements of $A$. Under $\mathrm{CH}$, then each $\alpha$ in $A$ is preceded by countably many elements of $A$. Let $\alpha \in A$. According to the Baire Category Theorem, $\cap\left\{A_{\beta}: \beta \leq \alpha\right\} \in \mathcal{D}$. The $x$-projection $\Pi_{1}\left(K_{\alpha}\right)$ of $K_{\alpha}$ being a nondegenerate interval $[a, b][4]$, there is by transfinite induction a Cantor set $C_{\alpha}$ disjoint from $\cup\left\{C_{\beta}: \beta<\alpha\right\}$ such that

$$
C_{\alpha} \subset[a, b] \cap\left(\cap\left\{A_{\beta}: \beta \leq \alpha\right\}\right) .
$$

For each $x \in C_{\alpha}$, define

$$
g(x)=\max \Pi_{2}\left(K_{\alpha} \cap(\{x\} \times I)\right),
$$

where $\Pi_{2}$ denotes the $y$-projection. Then $g_{\mid C_{\alpha}}$ is upper semicontinuous, therefore in Baire class 1, and consequently Marczewski measurable, which means that a perfect set like $C_{\alpha}$ has a perfect subset $P_{\alpha}$ such that $g_{\mid P_{\alpha}}$ is continuous. For argument's sake, we may suppose $g\left(P_{\alpha}\right)$ is nowhere dense. For, if $g\left(P_{\alpha}\right)$ contains an interval $[c, d]$ and if $D$ is a Cantor set in $[c, d]$, then the closed set $g^{-1}(D) \cap P_{\alpha}$ has $c$-many points and therefore contains a Cantor set $P$. So $g(P)$ is nowhere dense as desired. Define $f=g$ on $P_{\alpha}$ for each $\alpha \in A$, and define

$$
f\left(I \backslash \cup\left\{P_{\alpha}: \alpha \in A\right\}\right)=0 .
$$

By construction, $f: I \rightarrow I$ is dense in $I^{2}$ and almost continuous because $f$ meets every blocking set of $I^{2}$.

We show $f$ has the SCIVP. Suppose $E$ is a Cantor set between different values $f(x)$ and $f(y)$ with $x<y$, and let $I_{1}, I_{2}, I_{3}, \ldots$ be the interval components of $I \backslash E$. A nondecreasing continuous function $h: I \rightarrow[x, y]$ can be constructed like the Cantor ternary function so that $h$ is a different constant $r_{n}$ on each $I_{n}$. Then the inverse relation

$$
h^{-1}=\{(r, s):(s, r) \in h\} \in \mathcal{X}
$$

implies $h^{-1}=K_{\alpha_{o}}$ for some $\alpha_{o} \in A$, and so $f_{\mid P_{\alpha_{o}}} \subset K_{\alpha_{o}}$ and is continuous. $P_{\alpha_{o}} \backslash\left\{r_{1}, r_{2}, r_{3}, \ldots\right\}$ contains a Cantor set $B$ between $x$ and $y$; moreover $f(B) \subset$ $E$ and $f_{\mid B}$ is continuous. 
Assume $f$ is extendable. There exists a dense $G_{\delta^{-}}$-subset $G$ of $I$ that is $f$-negligible [7]; i.e., no matter how $f$ is arbitrarily redefined on $G$ with values in $I$, the resulting function is still extendable. Since $G \in \mathcal{D}, G=A_{\alpha_{1}}$, for some $\alpha_{1} \in A$. Let

$$
\left\{P_{\alpha}: \alpha<\alpha_{1}\right\}=\left\{P_{1}, P_{2}, P_{3}, \ldots\right\} .
$$

We may redefine $f=0$ on $A_{\alpha_{1}}$ and the resulting function $f$ is still extendable. Since $P_{\alpha} \subset A_{\alpha_{1}}$ for all $\alpha \geq \alpha_{1}$, then

$$
f(I)=f\left(I \backslash \cup_{i=1}^{\infty} P_{i}\right) \cup\left(\cup_{i=1}^{\infty} f\left(P_{i}\right)\right)=\{0\} \cup\left(\cup_{i=1}^{\infty} f\left(P_{i}\right)\right),
$$

which is a union of countably many nowhere dense subsets of the nondegenerate interval $f(I)$. Contradiction.

Question 1. Without $\mathrm{CH}$ in Theorem 1, does there exist such a function in ZFC?

Question 2. With $\mathbb{R}$ replacing $I$ in the above definitions, does there exist an almost continuous nonextendable function $f: \mathbb{R} \rightarrow \mathbb{R}$ having the SCIVP and obeying $f(x+y)=f(x)+f(y)$ for all $x, y \in \mathbb{R}$ ?

Let $\mathcal{C}$ denote a class of functions $f: I \rightarrow I$, and let $E$ be a subset of $I$. $E$ is stationary for $\mathcal{C}$ if whenever $f \in \mathcal{C}$ and $f$ is constant on $E$, then $f$ is constant on all of $I$.

Theorem 2. If $E$ is stationary for the class $\mathcal{C}$ of extendable functions $f: I \rightarrow$ $I$, then $E$ intersects each dense $G_{\delta}$-subset of $I$.

Proof. Assume there is a stationary set $E$ for $\mathcal{C}$ that misses a dense $G_{\delta}$-subset $A_{\alpha}$ of $I$. There exists an extendable function $f: I \rightarrow I$ whose graph is dense in $I^{2}$ [1]. Let $A$ be a dense $G_{\delta}$-subset of $(0,1)$ that is $f$-negligible. $A_{0}=A \cap A_{\alpha}$ is a dense $G_{\delta}$-set that is $f$-negligible. By [6], there is a homeomorphism $h: I \rightarrow I$ such that $I \backslash A_{0} \subset h^{-1}\left(A_{0}\right)$ and $h^{-1}\left(A_{0}\right)$ is $(f \circ h)$-negligible. Since $E \subset I \backslash A_{0}$, $E$ is $(f \circ h)$-negligible. Therefore $f \circ h$ can be redefined just on $E$ to be 0 , but the resulting extendable function is not 0 on all of $I$, contrary to $E$ being stationary for $\mathcal{C}$.

According to [2], the next result holds for some other classes of functions, too.

Theorem 3. If a subset $E$ of I intersects each nonempty perfect subset of $I$, then $E$ is stationary for the class $\mathcal{C}$ of extendable functions $f: I \rightarrow I$. 
Proof. Suppose $f \in \mathcal{C}$ and $f$ is a constant $c$ on $E$. Assume there exists an $x \in I \backslash E$ such that $f(x) \neq c$. Since $f$ is extendable, $f$ has a perfect road at $x$ [3]. This means that there exists a perfect subset $P$ of $I$ containing $x$ and having $x$ as a bilateral limit point if $x$ is not an endpoint of $I$ such that $f_{\mid P}$ is continuous at $x$. Therefore there is a perfect subset $P_{0}$ of $P$ containing $x$ such that $c \notin f\left(P_{0}\right)$. But $E \cap P_{0} \neq \emptyset$ implies $c \in f\left(P_{0}\right)$, a contradiction. So $f$ equals $c$ on $I$, and $E$ is stationary for $\mathcal{C}$.

\section{References}

[1] J. B. Brown, Totally discontinuous connectivity functions, Colloq. Math. 23 (1971), 53-60.

[2] A. M. Bruckner and J. L. Leonard, Stationary sets and determining sets for certain classes of Darboux functions, Proc. Amer. Math. Soc., 16 (1965), 935-940.

[3] R. G. Gibson and F. Roush, Connectivity functions with a perfect road, Real Analysis Exchange, 11 (1985-86), 260-264.

[4] K. R. Kellum and B. D. Garrett, Almost continuous real functions, Proc. Amer. Math. Soc., 33 (1972), 181-184.

[5] J. Nash, Generalized Brouwer Theorem, Research Problem, Bull. Amer. Math. Soc., 62 (1956), 76.

[6] T. Natkaniec, Extendability and almost continuity, Real Analysis Exchange, 21 (1995-96), 349-355.

[7] H. Rosen, Limits and sums of extendable connectivity functions, Real Analysis Exchange, 20 (1994-95), 183-191.

[8] H. Rosen, R. G. Gibson, and F. Roush, Extendable functions and almost continuous functions with a perfect road, Real Analysis Exchange, 17 (1991-92), 248-257. 